\title{
QUALITY OF LIFE IN ADULT PATIENTS WITH SYSTEMIC AUTOIMMUNE MYOPATHIES: TOWARDS THE PATIENT-CENTERED CARE
}

\author{
Rafael Alves Cordeiro, ${ }^{1,}$, Dechristian França Barbieri², Frida Marina Fischer ${ }^{1}$, Samuel Katsuyuki Shinjo ${ }^{1}$ \\ 1. Universidade de São Paulo, São Paulo (SP), Brazil. 2. Universidade Federal de São Carlos, São Carlos (SP), Brazil. \\ *Corresponding author: rafael19abc@hotmail.com
}

\section{BACKGROUND}

There are few studies that have assessed patient-reported outcomes for patients with systemic autoimmune myopathies (SAMs). Therefore, the present study aimed to document self-perceived quality of life (QoL) and to identify factors associated with worse QoL in adult patients with SAMs.

\section{MATERIALS AND METHODS}

This is a unicenter, cross-sectional study that included adult patients with SAMs (2017 EULAR/ACR classification criteria) from 2019 to 2020. The QoL was ascertained via the World Health Organization Quality of Life (WHOQOL-BREF) tool. The WHOQOL-BREF total score (0-100 scale) and the scores of its four domains (physical, psychological, social relationships, and environment) were considered as dependent variables. The following data were also collected: sociodemographic information, myositis subgroup, disease duration, extramuscular manifestations, current prednisone dose, physician's visual analog scale (VAS) for disease activity, muscle strength (MMT-8), mass body index, serum creatine phosphokinase levels, patient's VAS (disease activity and pain), functionality (HAQ-DI), fatigue (FSS), and mental health (DASS-21). The t-test/ANOVA and linear regression ( $r$ correlation) were applied for the analysis of categorical and continuous variables, respectively. P-value $<0.05$ was considered significant.

\section{RESULTS}

A total of 75 patients with SAMs were enrolled, consisting of antisynthetase syndrome (37.3\%), clinically amyopathic dermatomyositis (25.3\%), dermatomyositis (22.7\%), immune-mediated necrotizing myopathy (8.0\%), and polymyositis (6.7\%). The mean age was $44.1 \pm 9.4$ years, with predominance of female (66.7\%) and non-Caucasian (70.7\%). The disease duration was $6.2 \pm 4.5$ years. The highest and the lowest mean scores of WHOQOL-BREF domains were social relationships (mean: 67.1 \pm 20.5 ) and physical health (mean: $50.1 \pm 20.4$ ), respectively. The total QoL score was significantly lower in patients with the following features: female, history of arthritis, fatigue, depression, anxiety, and stress. In the linear regression analysis, a moderate negative correlation was observed between total QoL and physician's VAS ( $r=-0.44)$, patient's VAS $(r=-0.57)$, VAS for pain $(r=-0.69)$ and HAQ $(r=-0.59)$, all $p<0.001$. Regarding the specific domains of WHOQOL-BREF, female gender was statistically associated with lower scores in physical, psychological and environmental components. Fatigue, depression, and stress were associated with worse performances in all domains.

\section{CONCLUSION}

Incorporation of patients' perspective into clinical care and research may be used to address their unmet needs. According to our data, an association was found between female sex, mental health parameters, fatigue and history of arthritis with lower total QoL scores. The differences observed warrant further research, especially into patterns of QoL changes after therapy with emphasis on mental health, fatigue, and pain. 